\title{
Temporal Coding Mediates Discrimination of "Bitter" Taste Stimuli by an Insect
}

\author{
John I. Glendinning, Adrienne Davis, and Meelu Rai \\ Department of Biological Sciences, Barnard College, Columbia University, New York, New York 10027
}

The mechanisms that mediate discriminative taste processing in insects are poorly understood. We asked whether temporal patterns of discharge from the peripheral taste system of an insect (Manduca sexta caterpillars; Sphingidae) contribute to the discrimination of three "bitter" taste stimuli: salicin, caffeine, and aristolochic acid. The gustatory response to these stimuli is mediated exclusively by three pairs of bitter-sensitive taste cell, which are located in the medial, lateral, and epipharyngeal sensilla. We tested for discrimination by habituating the caterpillars to salicin and then determining whether the habituation generalized to caffeine or aristolochic acid. We ran habituation-generalization tests in caterpillars with their full complement of taste sensilla (i.e., intact) and in caterpillars with ablated lateral sensilla (i.e., lat-ablated). The latter perturbation enabled us to examine discrimination in caterpillars with a modified peripheral taste profile. We found that the intact and lat-ablated caterpillars both generalized the salicin-habituation to caffeine but not aristolochic acid. Next, we determined whether this pattern of stimulus-generalization could be explained by salicin and aristolochic acid generating distinct ensemble, rate, temporal, or spatiotemporal codes. To this end, we recorded excitatory responses from the bitter-sensitive taste cells and then used these responses to formulate predictions about whether the salicin-habituation should generalize to caffeine or aristolochic acid, separately for each coding framework. We found that the pattern of stimulus generalization in both intact and latablated caterpillars could only be predicted by temporal coding. We conclude that temporal codes from the periphery can mediate discriminative taste processing.

Key words: taste; bitter; temporal coding; discrimination; insect; Manduca sexta

\section{Introduction}

There is a long-standing debate about how animals identify and discriminate taste stimuli. Proponents of labeled-line coding contend that tastant identity is represented by activity in a subset of afferent gustatory neurons (Hellekant et al., 1997; Zhang et al., 2003; Marella et al., 2006), whereas proponents of ensemble coding contend that tastant identity is represented by activity across large populations of afferent gustatory neurons (Dethier and Crnjar, 1982; Erickson et al., 1995; Caicedo et al., 2002). We believe that the persistence of this debate reflects the fact that these coding frameworks fail to capture the full complexity of taste processing. Indeed, there is evidence that rate (Scott and Perrotto, 1980; Scott and Giza, 1987), temporal (Katz et al., 2002a), and spatiotemporal (Verhagen and Scott, 2004) codes contribute to taste processing. Here, we provide evidence that temporal codes from taste cells can mediate the discrimination of "bitter" taste stimuli.

Temporal codes in the taste system are represented by differences in spike timing, spike synchronicity, or firing rate dynamics

Received March 9, 2006; revised July 17, 2006; accepted July 24, 2006.

This work was supported in part by National Institute on Deafness and Other Communication Disorders-National Institutes of Health Research Grant 5 R29 DC 02416 (J.I.G.) and by a grant from the Howard Hughes Medical Institute (Barnard College). We thank Peter Balsam, Patricia Di Lorenzo, and Don Katz (and his students) for valuable editorial comments.

Correspondence should be addressed to John I. Glendinning, Department of Biological Sciences, Barnard College, Columbia University, 3009 Broadway, New York, NY 10027. E-mail: jglendinning@barnard.edu. DOI:10.1523/JNEUROSCI.2351-06.2006

Copyright $\odot 2006$ Society for Neuroscience $\quad$ 0270-6474/06/268900-09\$15.00/0
(Di Lorenzo and Victor, 2003; Katz, 2005). We asked whether Manduca sexta caterpillars use firing rate dynamics in discriminative taste processing. This insect's peripheral taste system consists of eight pairs of taste sensilla (Fig. 1a,c). Each sensillum contains three to four taste cells, which project directly to the CNS without synapsing. One taste cell in each sensillum responds selectively to compounds humans describe as bitter, whereas the other taste cells respond to nutrients. These taste cells exhibit low baseline activity $(<2 \mathrm{~Hz})$ (Städler and Hanson, 1975) but concentration-dependent increases in firing rate (Glendinning et al., 2002).

We demonstrated elsewhere that $M$. sexta caterpillars can discriminate between salicin, aristolochic acid, and caffeine (Glendinning et al., 2002). The gustatory response to these bitter taste stimuli is mediated exclusively by three pairs of bitter-sensitive taste cells, which are located in the medial, lateral, and epipharyngeal sensilla (Fig. 1c). Because salicin, caffeine, and aristolochic acid all activate the same taste cells, their discrimination could not involve labeled-line coding. Here, we assessed the potential contribution of four other coding frameworks to the discrimination: firing rates (rate coding), relative patterns of activity across taste cells (ensemble coding), discharge patterns (temporal coding), or temporally dynamic ensemble codes (spatiotemporal coding).

To test for discrimination, we used a habituation-generalization paradigm. This involved habituating the caterpillar's aversive response to salicin (Glendinning et al., 2001) and then determining 

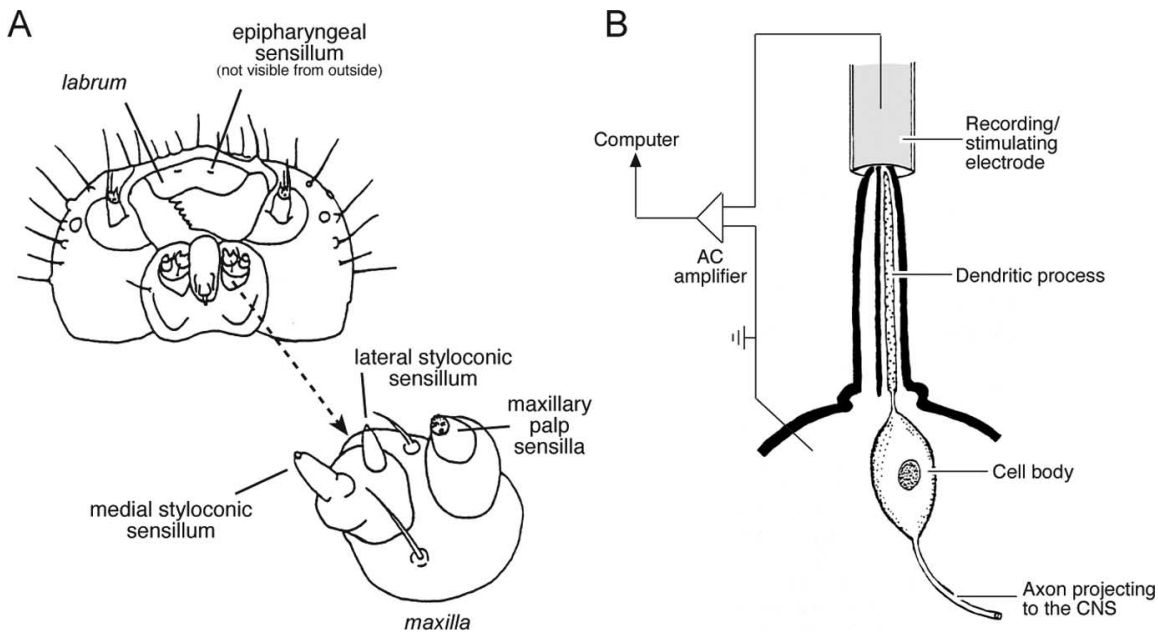

\begin{tabular}{|c|c|c|}
\hline $\begin{array}{l}\text { Class of } \\
\text { sensillum }\end{array}$ & $\begin{array}{l}\text { Identified taste cells } \\
\text { in each sensillum }\end{array}$ & Known ligands for each taste cell \\
\hline $\begin{array}{l}\text { Lateral styloconic } \\
(\mathrm{N}=1 \text { pair) }\end{array}$ & $\begin{array}{l}1 \text { (bitter-sensitive) } \\
2 \text { (inositol-sensitive) } \\
3 \text { (sugar-sensitive) } \\
4 \text { (salt-sensitive) }\end{array}$ & $\begin{array}{l}\text { aristolochic acid, caffeine, salicin } \\
\text { inositol } \\
\text { glucose, sucrose } \\
\mathrm{NaCl}, \mathrm{KCl}\end{array}$ \\
\hline $\begin{array}{l}\text { Medial styloconic } \\
(\mathrm{N}=1 \text { pair) }\end{array}$ & $\begin{array}{l}1 \text { (bitter-sensitive) } \\
2 \text { (inositol-sensitive) } \\
3 \text { (sugar-sensitive) } \\
4 \text { (salt-sensitive) }\end{array}$ & $\begin{array}{l}\text { aristolochic acid, caffeine, salicin, Canna extract } \\
\text { inositol } \\
\text { glucose } \\
\mathrm{NaCl}, \mathrm{KCl}\end{array}$ \\
\hline $\begin{array}{l}\text { Epipharyngeal } \\
(\mathrm{N}=1 \text { pair })\end{array}$ & $\begin{array}{l}1 \text { (bitter-sensitive) } \\
2 \text { (acid \& salt-sensitive) } \\
3 \text { (acid-sensitive) }\end{array}$ & $\begin{array}{l}\text { aristolochic acid, caffeine, salicin, Canna extract } \\
\text { oxalic acid, } \mathrm{NaCl}, \mathrm{KCl} \\
\text { oxalic acid }\end{array}$ \\
\hline $\begin{array}{l}\text { Maxillary palp } \\
(\mathrm{N}=5 \text { pairs })\end{array}$ & $\begin{array}{l}1 \text { (bitter-sensitive) } \\
2 \text { (salt-sensitive) } \\
3 \\
4\end{array}$ & $\begin{array}{l}\text { Grindelia extract } \\
\mathrm{NaCl}, \mathrm{KCl} \\
--\end{array}$ \\
\hline
\end{tabular}

Figure 1. A, Diagram of the head of a M. sexta caterpillar, as viewed from below. An enlargement of the maxilla (indicated with an arrow) is provided to clarify the location of the medial and lateral styloconic sensilla. The epipharyngeal sensilla are located underneath the labrum and thus are not visible in this diagram. This diagram was adapted from Bernays and Chapman (1994, their Fig. 3.4). $\boldsymbol{B}$, Illustration of the tip recording method, which was used to record excitatory responses of individual taste cells located within a taste sensillum. During a tip recording (Hodgson et al., 1955), the tip of a taste sensillum is inserted into the end of a glass recording/stimulating electrode, which is filled with a taste stimulus dissolved in an electrolyte solution $(0.1 \mathrm{~m} \mathrm{KCl}$ in deionized water). The taste stimulus solution diffuses through a pore in the tip of the sensillum and activates transduction mechanism(s) on the distal end of the dendritic process of a taste cell; the electrode detects the ensuing action potentials. For clarity, only one taste cell is indicated. Note that the axonal process of the taste cell projects directly to the CNS without synapsing. C, Table illustrating the known ligands for taste cells within the lateral styloconic, medial styloconic, epipharyngeal, and maxillary palp sensilla. There are four taste cells in each of the lateral and medial styloconic sensilla, three taste cells in each of the epipharyngeal sensilla, and four taste cells in each of the taste sensilla on the maxillary palp. One taste cell in each taste sensillum is bitter sensitive (Schoonhoven, 1972; de Boer et al., 1977; Glendinning et al., 2002). Note that the bitter-sensitive taste cells in the lateral, medial, and epipharyngeal sensilla each respond to salicin, caffeine, and aristolochic acid (Glendinning and Hills, 1997; J. Glendinning, unpublished data). Despite limited knowledge of the molecular receptive range of the bitter-sensitive taste cells in the maxillary palp sensilla, we know that none of them respond to caffeine, salicin, or aristolochic acid (Glendinning et al., 1998).

whether the habituation generalized to caffeine or aristolochic acid. For instance, if it generalized to caffeine, then we inferred that the caterpillars could not discriminate salicin and caffeine. If it failed to generalize to caffeine, then we inferred that the caterpillars could discriminate salicin and caffeine. We hypothesized that the caterpillars habituate specifically to the decelerating firing rate that salicin elicits in the bitter-sensitive taste cells (Glendinning and Hills, 1997). Accordingly, the salicin-habituation should generalize to those bitter taste stimuli that generate a de- celerating firing rate (caffeine) but not to ones that generate an accelerating firing rate (aristolochic acid).

\section{Materials and Methods \\ Subjects and rearing conditions}

The caterpillars were reared from eggs on a wheat germ-based artificial diet (Bell and Joachim, 1976) and were maintained in an environmental chamber with a $16 / 8 \mathrm{~h}$ light/dark cycle at $25^{\circ} \mathrm{C}$. We began all experiments with caterpillars during the second day of their fifth larval instar. All caterpillars were naive to the taste stimuli before testing. To control for potential differences among caterpillars from different egg batches, individuals from each batch were interspersed randomly across treatment levels according to a blind procedure. We indicate sample sizes in the figure legends.

\section{Habituation protocol}

We provided a detailed description of the habituation protocol previously (Glendinning et al., 2001). In brief, we offered the "salicinhabituated" caterpillars a block of the rearing diet, which had been adulterated with a $6 \%$ concentration (wet mass) of salicin ( $=157 \mathrm{~mm}$ $\mathrm{kg}^{-1}$ diet); this salicin diet was their only source of food and water for $24 \mathrm{~h}$. Over the course of this exposure period, the caterpillars become completely habituated to salicin concentrations as high as $12 \%$ (Glendinning et al., 2001). The "nonhabituated" caterpillars received a salicinfree block of the same diet adulterated with $6 \%$ (wet mass) alphacel (an indigestible form of cellulose; ICN Biomedicals, Cleveland, $\mathrm{OH}$ ); this diet was their only source of food and water for $24 \mathrm{~h}$. We know that the habituation protocol attenuates the aversive response through a central gustatory mechanism, because the bittersensitive taste cells of both salicin-habituated and nonhabituated caterpillars respond equally vigorously to salicin (Glendinning et al., 2001).

\section{Experiment 1: how do the bitter-sensitive} taste cells respond to salicin, caffeine, and aristolochic acid in salicin-habituated caterpillars?

Several electrophysiological studies have shown that the bitter-sensitive taste cells in the lateral styloconic, medial styloconic, and epipharyngeal sensilla of $M$. sexta respond to aristolochic acid with an accelerating discharge pattern and to salicin and caffeine with a decelerating discharge pattern (Frazier, 1986; Glendinning and Hills, 1997; Glendinning et al., 1998). These different discharge patterns are thought to reflect the presence of two transduction pathways in each of the bitter-sensitive taste cells: one responds to aristolochic acid and the other responds to caffeine and salicin (Glendinning and Hills, 1997; our unpublished data). Accordingly, the transduction pathway for aristolochic acid takes $\sim 1.0 \mathrm{~s}$ to reach peak activity, resulting in the accelerating discharge pattern; in contrast, the transduction pathway for caffeine and salicin takes $\sim 0.05 \mathrm{~s}$ to reach peak activity and then begins to adapt almost immediately, resulting in the decelerating discharge pattern.

Because the previous studies of discharge patterns used nonhabituated caterpillars, we repeated them here with salicin-habituated caterpillars. Our goal was to describe the discharge patterns of the bitter-sensitive 
taste cells in the lateral styloconic, medial styloconic, and epipharyngeal sensilla when stimulated with a range of concentrations of salicin, caffeine, and aristolochic acid.

Taste stimuli. In all experiments, the taste solutions were prepared immediately before testing and were presented at room temperature. The taste stimuli were purchased from Sigma-Aldrich (St. Louis, MO). Here, we tested three concentrations of salicin $(10,30,100 \mathrm{~mm})$, caffeine $(1,3$, $10 \mathrm{~mm})$, and aristolochic acid sodium salt $(0.03,0.06,0.1 \mathrm{~mm})$; these concentrations were selected based on previous studies (Glendinning et al., 1999). We dissolved the taste stimuli in a $0.1 \mathrm{M} \mathrm{KCl}$ solution.

Electrophysiological recordings. We recorded responses of individual taste cells using a noninvasive extracellular tip-recording technique (Fig. 1b) (Gothilf and Hanson, 1994; Glendinning et al., 1998). In brief, we placed a glass electrode containing a taste stimulus solution over the tip of a lateral or medial styloconic sensillum or directly on top of an epipharyngeal sensillum (after deflecting the labrum back $90^{\circ}$ from its normal position) (de Boer et al., 1977; Glendinning et al., 1999). We recorded extracellular AC signals with the Taste-Probe amplifier system (Syntech, Hilversum, The Netherlands) (Marion-Poll and Van der Pers, 1996). We preamplified each recording 10 times, ran it through a bandpass filter set at $100-1200 \mathrm{~Hz}$, fed it into a computer through a 16-bit analog-to-digital converter board, and then analyzed it off-line with Autospike software (Syntech).

Because each taste sensillum contains three to four taste cells, a taste stimulus solution could potentially stimulate more than one taste cell. We identified spikes from the bitter-sensitive taste cells by relying on the fact that individual taste cells discharge in a regular temporal pattern. This is manifested as interspike intervals that gradually decrease (or increase) over time in an orderly manner (White et al., 1990; Bernays et al., 2000; Glendinning et al., 2002). Using this spike identification procedure, it was apparent that the response of individual sensilla to a bitter taste stimulus (e.g., $5 \mathrm{~mm}$ caffeine) consisted predominantly of spikes from a single bitter-sensitive taste cell (see Fig. 2a). In some (but not all) traces, there were a few sporadic spikes occurring out of phase with those from the bitter-sensitive taste cell. These latter spikes reflect the response of the salt-sensitive taste cell to $0.1 \mathrm{KCl}$, which was added to each taste solution for electrical conductivity. Whenever there was ambiguity about spike assignment based on discharge patterns, we used the relative height and shape of spikes as distinguishing features. We quantified the neural response of each bitter-sensitive taste cell by tallying the number of spikes that occurred during each successive $0.1 \mathrm{~s}$ time bin.

To minimize carry-over effects between recordings, we paused at least 3 min between stimulations. To minimize solvent evaporation at the tip of the recording/stimulating electrode, we drew fluid from the tip with a piece of filter paper immediately before stimulation. To minimize any order effects, we randomized the order of stimulus presentation. Finally, to ensure that our neural recordings were independent (in a statistical sense), we stimulated only one taste sensillum per caterpillar.

Data analysis. Our goal was to determine whether the bitter-sensitive taste cells of salicin-habituated caterpillars responded to each concentration of aristolochic acid with an accelerating discharge pattern and to each concentration of caffeine and salicin with a decelerating discharge pattern. To this end, we used a one-way repeated-measure ANOVA to analyze the discharge pattern (i.e., number of spikes during each of 10 successive $100 \mathrm{~ms}$ time bins), separately for each taste stimulus and concentration. The main effect in each ANOVA was time. In this and all subsequent experiments, we set the $\alpha$ level at 0.05 .

\section{Experiment 2: which sensory code could mediate discrimination?} This experiment had two goals. The first was to describe the peripheral gustatory response of salicin-habituated caterpillars to approximate isostimulatory concentrations of salicin, caffeine, and aristolochic acid. We focused on the initial $0.5 \mathrm{~s}$ of neural response because results presented below (see experiment 3 ) show that it is the operative time frame for the discrimination.

The second goal of this experiment was to use the peripheral gustatory responses to formulate predictions about whether the salicinhabituation should generalize to caffeine or aristolochic acid, separately for each coding framework. We formulated two sets of predictions for each coding framework: one for caterpillars with their full complement of taste sensilla (i.e., intact caterpillars) and another for caterpillars lacking their lateral sensilla (i.e., lat-ablated caterpillars). Because eliminating sensory input from the lateral sensilla substantially altered the peripheral gustatory response, the predictions for intact and lat-ablated caterpillars differed. By testing two different sets of predictions for each coding framework, we reduced the probability that an agreement between the predicted and observed pattern of habituation-generalization could be explained by chance alone.

Taste stimuli. We examined the neural response to a single concentration of each bitter taste stimulus. We selected $100 \mathrm{~mm}$ salicin, because it elicits the maximal response to salicin in all three bitter-sensitive taste cells (Glendinning et al., 1999); this maximal response should match the neural response generated by the habituating diet, which contained a salicin concentration of $157 \mathrm{~mm} / \mathrm{kg}$ diet (fresh mass). We selected $5 \mathrm{~mm}$ caffeine and $0.03 \mathrm{~mm}$ aristolochic acid because previous studies indicated that these concentrations were approximately isostimulatory with the $100 \mathrm{~mm}$ concentration of salicin in nonhabituated (Glendinning et al., 1999) and habituated (see experiment 2) caterpillars. The $\mathrm{pH}$ of all three taste stimuli was 5.1.

Electrophysiological recordings. We examined the responses of each bitter-sensitive taste cell using the same electrophysiological and spike identification procedures described in the previous experiment. We stimulated one taste sensillum per caterpillar with each of the three bitter taste stimuli (in a randomized order) and then quantified responses of the bitter-sensitive taste cells during the initial $0.5 \mathrm{~s}$ of response. To simulate the response of lat-ablated caterpillars, we simply excluded responses from the bitter-sensitive taste cell in the lateral sensillum. This "virtual ablation" approach was justified by the fact that unpublished studies in our laboratory have shown that ablating the lateral styloconic sensilla does not alter the responsiveness of the bitter-sensitive taste cells in the other taste sensilla.

Data analysis. To formulate predictions about the pattern of habituation-generalization, we compared the neural responses to (1) salicin and caffeine and then (2) salicin and aristolochic acid. Because we ran separate comparisons for the intact and lat-ablated caterpillars, we could generate a unique set of predictions for each type of caterpillar.

To test for a rate or ensemble code, we analyzed the total number of spikes generated by each class of bitter-sensitive taste cells during the initial $0.5 \mathrm{~s}$ of stimulation, using a two-way ANOVA. We treated the taste cell as a between factor and taste stimulus as a within factor. We inferred a difference in rate code (e.g., between salicin and caffeine) if there was a significant main effect of taste stimulus. We inferred a difference in ensemble code if there was a significant interaction of taste stimulus $x$ taste cell.

To test for a temporal code, we examined the temporal dynamics of response during the initial $0.5 \mathrm{~s}$ of stimulation. To this end, we obtained time-intensity (T-I) curves (i.e., number of spikes during each successive $0.1 \mathrm{~s}$ time bin), separately for each taste stimulus and bitter-sensitive taste cell. We analyzed these T-I curves with a three-way ANOVA, treating time and taste cell as between factors and taste stimulus as a within factor. We inferred a difference in temporal code between two taste stimuli (e.g., salicin and caffeine) if the interaction of time $x$ taste stimulus was significant.

To test for a spatiotemporal code, we analyzed the T-I curves with a two-way ANOVA, treating time as a within factor and taste cell as a between factor. We analyzed the results from each taste stimulus separately and asked how the T-I curves from each class of bitter-sensitive taste cell changed relative to one another over the initial $0.5 \mathrm{~s}$ of response. We inferred a difference in spatiotemporal code between two taste stimuli (e.g., salicin and caffeine) if the T-I curves from one taste stimulus produced a significant interaction of time $x$ taste cell, whereas those from another taste stimulus produced a nonsignificant interaction of time $x$ taste cell.

\section{Experiment 3: which coding framework best explains the pattern} of stimulus generalization?

In the previous experiment, we formulated specific predictions about the pattern of habituation-generalization based on responses of the bitter- 
sensitive taste cells. Here, we evaluated these predictions by measuring the initial biting response of salicin-habituated caterpillars to disks treated with water, $5 \mathrm{~mm}$ caffeine, or $0.03 \mathrm{~mm}$ aristolochic acid. We determined the pattern of stimulus generalization in both intact and in lat-ablated caterpillars. As a positive control, we also measured the initial biting response of nonhabituated caterpillars to disks treated with water, $5 \mathrm{~mm}$ caffeine, or $0.03 \mathrm{~mm}$ aristolochic acid. We expected the nonhabituated caterpillars to exhibit an aversive response to the caffeine and aristolochic acid disks but not the water disk.

Taste stimuli. Taste stimuli were presented to caterpillars in a glassfiber disk ( $4.25 \mathrm{~cm}$ diameter; Whatman GF/A; Whatman International, Maidstone, UK). Immediately before a test, we pipetted $400 \mu \mathrm{l}$ of taste stimulus (dissolved in deionized water) onto a disk and then offered it to a caterpillar. We treated the inositol disk (see below) with $10 \mathrm{~mm}$ myoinositol, because it is known to stimulate biting in M. sexta (Glendinning et al., 2000). We treated the experimental disks (see below) with $5 \mathrm{~mm}$ caffeine, $0.03 \mathrm{~mm}$ aristolochic acid, or deionized water alone (hereafter, "control").

Biting test. We conducted the biting test in two separate test arenas, each of which consisted of an inverted Petri dish with a piece of fresh cork $(1 \mathrm{~cm}$ diameter, $0.4 \mathrm{~cm}$ tall) attached to the center. Because each test arena was positioned on a turntable-like device, we could rotate the caterpillar as it fed and keep its mandibles clearly visible.

After a $30 \mathrm{~min}$ period of food deprivation, the caterpillar was placed in Test Arena 1 with the inositol disk pinned to the cork. Care was taken to ensure that the caterpillar's legs and prolegs grasped the inositol disk. Once the caterpillar began to bite the inositol disk (but before it had taken $>30$ bites), it was transferred to one of the experimental disks. To minimize disturbance during this transfer, we positioned the experimental disk next to the caterpillar's head and then waited for the caterpillar to walk onto the experimental disk and grasp its edge. Then, we transferred the experimental disk (with caterpillar attached) to Test Arena 2 and pinned the disk to the cork. We considered the biting test to have begun once the caterpillar took its first bite from the experimental disk and then terminated the biting test 1 min later. We inferred that the caterpillar was not disturbed by the transfer from the inositol to the experimental disk if it took $\geq 20$ bites from the experimental disk during the test. However, if the caterpillar took $<20$ bites on the experimental disk, then we did not know whether the low biting activity was caused by the transfer procedure or the aversive taste stimulus. To distinguish between these two possibilities, we transferred the caterpillar back to the inositol disk immediately after the $1 \mathrm{~min}$ trial. If it reinitiated vigorous biting on the inositol disk (i.e., took $>20$ bites in $<30$ s), then we inferred that the aversive taste stimulus inhibited biting. If the caterpillar failed to initiate biting on the inositol disk, then we inferred that the transfer procedure inhibited biting. We included in the analysis only those caterpillars that either took $>20$ bites from the experimental disk during the $1 \mathrm{~min}$ trial or that reinitiated vigorous biting on the inositol disk immediately after the 1 min trial. Using these criteria, we included $94 \%$ of the caterpillars.

Throughout the 1 min test, a trained observer recorded the timing of each bite with a software-based event recorder. Because each caterpillar took bites at a low frequency $(0-3 \mathrm{~Hz})$, and because the caterpillars' black mandibles contrasted with the white experimental disks, the trained observer was able to record the timing of each bite easily and accurately. To minimize observer bias, we kept the observer blind to the identity of the taste stimulus in the experimental disk (i.e., water, aristolochic acid, or caffeine) and randomized the presentation order of the experimental disks.

After the first biting test, we transferred the caterpillar back to its rearing diet and permitted it to feed ad libitum for $30 \mathrm{~min}$. Then, we food-deprived the caterpillar for $30 \mathrm{~min}$ and ran it through the next biting test with a different experimental disk. Each caterpillar was subjected to three biting tests, each with a different experimental disk.

Sensilla ablation procedure. We used an established procedure for ablating the bilateral pair of lateral styloconic sensilla (de Boer and Hanson, 1987). In brief, we put a recently molted fifth-instar caterpillar on ice for $30 \mathrm{~min}$, secured its head with a rubber gasket, and then removed the two lateral styloconic sensilla by snipping each of them near their base with iridectomy scissors. Immediately after the ablation, we returned the cat- erpillar to its rearing diet for $23 \mathrm{~h}$. All of the caterpillars survived the ablation procedure without any apparent complications and were subjected to the habituation-generalization test within $24 \mathrm{~h}$ of the procedure.

Data analysis. We limited our data analysis to the initial $5 \mathrm{~s}$ of biting. This approach was made possible by the observation that the repetitive biting sequence of $M$. sexta is disrupted almost immediately by aversive gustatory input, resulting in longer and more variable interbite intervals (IBIs) (Dethier and Crnjar, 1982; Frazier, 1986).

We calculated two dependent measures: (1) the latency between the first and second bite (i.e., the initial interbite interval), and (2) biting activity during the initial $5 \mathrm{~s}$ of biting (i.e., the number of bites taken during each successive $1 \mathrm{~s}$ time bin). If the initial IBI on the caffeine or aristolochic acid disk was significantly longer than that on the control disk, then we inferred that the taste stimulus elicited an aversive response. We also compared the number of bites taken during each of the $1 \mathrm{~s}$ time bins. We used the paired $t$ test to make all of these comparisons. To control for the use of multiple $t$ tests, we used Bonferroni corrections (i.e., we divided the $\alpha$ level by the number of $t$ test comparisons).

\section{Results}

\section{Experiment 1: how do the bitter-sensitive taste cells respond to salicin, caffeine, and aristolochic acid in salicin-habituated caterpillars?}

In Figure $2 a$, we show typical neural responses from the lateral styloconic sensillum. These responses illustrate three distinctive features of the peripheral gustatory response. First, one taste cell (the salt-sensitive taste cell) generated infrequent and intermittent spikes in response to the solvent alone (i.e., $0.1 \mathrm{M} \mathrm{KCl}$ ). Second, a single bitter-sensitive taste cell discharged regularly and rapidly in response to each bitter taste stimulus. Third, the bittersensitive taste cell responded to aristolochic acid with an accelerating firing rate and to caffeine and salicin with a decelerating firing rate.

In Figure $2 b$, we quantify the discharge pattern for each class of bitter-sensitive taste cell to stimulation with several concentrations of caffeine, salicin, and aristolochic acid. In some bittersensitive taste cells, the lower concentrations of the bitter taste stimuli failed to elicit a response that could be distinguished from that to the solvent alone; we excluded these subthreshold concentrations from Figure $2 b$. All suprathreshold concentrations of aristolochic acid elicited a discharge pattern that accelerated significantly with time (all $F$ values $>21.2 ; n=7-13$ taste cells/ concentration; $p<0.01)$, whereas all suprathreshold concentrations of caffeine and salicin elicited a discharge pattern that decelerated significantly with time (all $F$ values $>12.4 ; n=7-13$ taste cells/concentration; $p<0.01$ ) (Fig. 2). Comparison of these findings with those published previously (Glendinning et al., $2001,2002)$ confirms that the salicin-habituation process did not alter the firing rate or distinctive discharge patterns generated by salicin, caffeine, and aristolochic acid.

In experiments 2 and 3, we used a single concentration of salicin $(100 \mathrm{~mm})$, aristolochic acid $(0.03 \mathrm{~mm})$, and caffeine (5 $\mathrm{mm}$ ). We selected these concentrations because they elicited approximately isostimulatory responses in the bitter-sensitive taste cells of the salicin-habituated caterpillars (Fig. 2).

\section{Experiment 2: which sensory code could mediate discrimination?}

We asked whether the responses of the bitter-sensitive taste cells to $100 \mathrm{~mm}$ salicin, $0.03 \mathrm{~mm}$ aristolochic acid, and $5 \mathrm{~mm}$ caffeine could be discriminated based on a rate, ensemble, temporal, or spatiotemporal code. We limited our analysis to the initial $0.5 \mathrm{~s}$ of response because the stimulus-generalization tests in experiment 3 (see below) indicated that the discrimination phenomenon 
occurs within this time frame. We present results from the intact and lat-ablated caterpillars separately.

\section{Intact caterpillars}

To test for distinct rate or ensemble codes, we examined the total number of spikes during the initial $0.5 \mathrm{~s}$ of response (Fig. $3 a$ ). In the salicin/caffeine comparison, there was a significant main effect of taste stimulus $\left(F_{(1,76)}=8.0 ; p \leq 0.05\right)$ but a nonsignificant interaction of taste stimulus $\times$ taste cell $\left(F_{(2,76)}=0.9 ; p>0.05\right)$. This indicates that salicin and caffeine generated different rate codes (i.e., caffeine $>$ salicin) but similar ensemble codes in the intact caterpillars. In the salicin/aristolochic acid comparison, there was a significant main effect of taste stimulus $\left(F_{(1,76)}=\right.$ $52.5 ; p \leq 0.05)$ and a significant interaction of taste stimulus $\times$ taste cell $\left(F_{(2,76)}=\right.$ $14.2 ; p \leq 0.05)$. This indicates that salicin and aristolochic acid generated different rate codes (i.e., salicin $>$ aristolochic acid) and ensemble codes in the intact caterpillars.

To test for distinct temporal codes, we ran a three-way ANOVA on the T-I curves (Fig. $3 b$ ) but focused exclusively on the two-way interaction of time $\times$ taste stimulus. In the salicin/caffeine comparison, the interaction of time $\times$ taste stimulus was nonsignificant $\left(F_{(4,112)}=1.9 ; p>\right.$ $0.05)$, showing that salicin and caffeine generated similar temporal codes (i.e., decelerating firing rates) in the intact caterpillars. In the salicin/aristolochic acid comparison, the interaction of time $\times$ taste stimulus was significant $\left(F_{(4,112)}=\right.$ $108.9 ; p \leq 0.05)$, revealing that salicin and aristolochic acid each generated different temporal codes (i.e., decelerating versus accelerating firing rates) in the intact caterpillars.

To test for distinct spatiotemporal codes, we analyzed the T-I curves from the three bitter-sensitive taste cells, separately for each taste stimulus (Fig. $3 b$ ). For salicin, there was a significant interaction of time $\times$ taste cell $\left(F_{(8,112)}=3.2 ; p \leq 0.05\right)$; this result, together with visual inspection of Figure $3 b$, reveals that salicin elicited nonparallel T-I curves in the three classes of bittersensitive taste cell. In contrast, for both caffeine and aristolochic acid, the interaction of time $\times$ taste cell was nonsignificant (in both cases, $p>0.05$ ); this result, together with visual inspection of Figure $3 b$, shows that caffeine and aristolochic acid each elicited T-I curves in the three classes of bitter-sensitive taste cell that were essentially parallel to one another. These findings indicate that salicin generated a different spatiotemporal code than caffeine and aristolochic acid in the intact caterpillars.

\section{Lat-ablated caterpillars}

In the next series of analyses, we re-examined the neural responses after excluding input from the bitter-sensitive taste cell in the lateral sensillum. To test for distinct rate or ensemble codes, we examined the total number of spikes across the initial $0.5 \mathrm{~s}$ of response (Fig. 3a). In the salicin/caffeine comparison, there was a
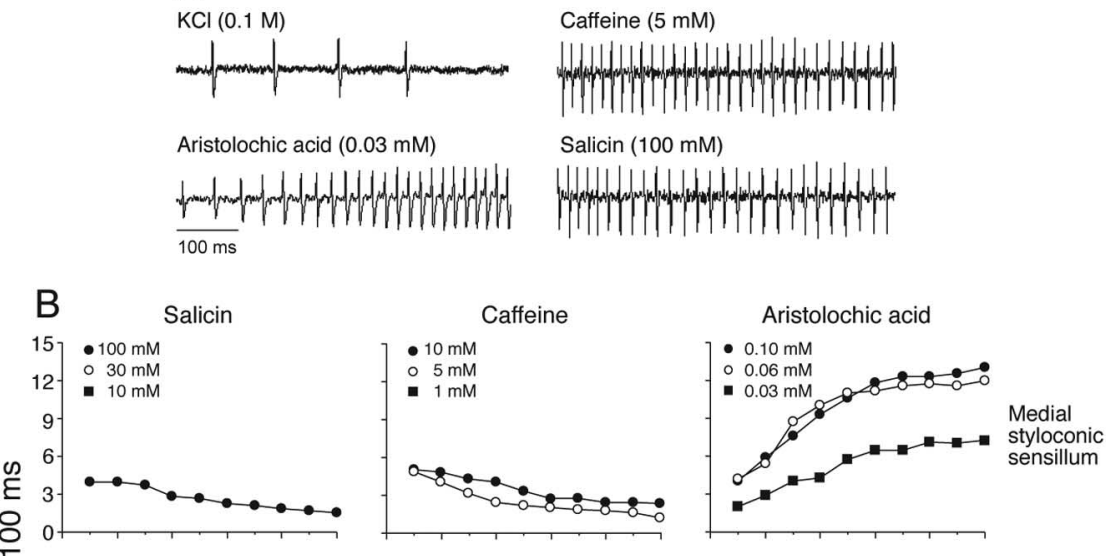

Figure 2. $A$, Typical neural responses of a lateral styloconic sensillum to $0.1 \mathrm{M} \mathrm{KCl}$ (the solvent for the other taste stimuli), 0.03 (middle bitter-sensitive taste cell in the medial styloconic (top row), epipharyngea (middle row), and lateral styloconic (bottom row) sensilla. We show responses of each bitter-sensitive taste cell to severa column of panels indicates the concentrations of each bitter taste stimulus that were used. All caterpillars were habituated to salicin before the recordings. $n=8-12$ bitter-sensitive taste cells per panel (each from a different caterpillar).

significant main effect of taste stimulus $\left(F_{(1,41)}=7.6 ; p \leq 0.05\right)$ but a nonsignificant interaction of taste stimulus $X$ taste cell $\left(F_{(1,41)}=0.2 ; p>0.05\right)$. This shows that salicin and caffeine each generated distinct rate codes (i.e., caffeine $>$ salicin) but similar ensemble codes in lat-ablated caterpillars. Likewise, in the salicin/ aristolochic acid comparison, there was a significant main effect of taste stimulus $\left(F_{(1,41)}=8.7 ; p \leq 0.05\right)$ and a nonsignificant interaction of taste stimulus $\times$ taste cell $\left(F_{(1,41)}=1.0 ; p>0.05\right)$. This reveals that salicin and aristolochic acid generated distinct rate codes (i.e., salicin $>$ aristolochic acid) but a similar ensemble codes in the lat-ablated caterpillars.

To test for distinct temporal codes, we ran a three-way ANOVA on the T-I curves (Fig. 3b), focusing on the two-way interaction of time $\times$ taste stimulus. In the salicin/caffeine comparison, the interaction of time $\times$ taste stimulus was nonsignificant $\left(F_{(4,60)}=1.9 ; p>\right.$ $0.05)$, showing that salicin and caffeine each generated similar temporal codes (i.e., decelerating firing rates) in the lat-ablated caterpillars. In the salicin/aristolochic acid comparison, however, the interaction of time $\times$ taste stimulus was significant $\left(F_{4,60}=97.8 ; p \leq\right.$ $0.05)$, revealing that salicin and aristolochic acid each generated distinct temporal codes (i.e., decelerating versus accelerating firing rates) in the lat-ablated caterpillars. 
A

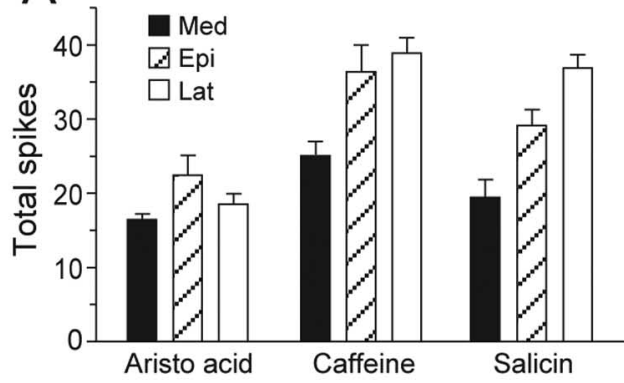

B

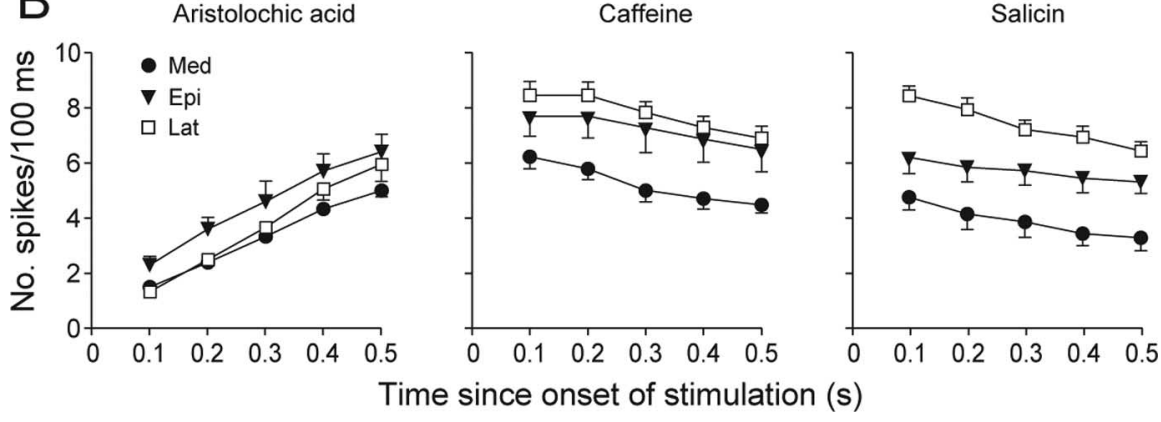

Figure 3. Neural responses of the bitter-sensitive taste cell in the medial styloconic (Med), epipharyngeal (Epi), and lateral styloconic (Lat) sensilla to $0.03 \mathrm{~mm}$ aristolochic acid (Aristo acid), $5 \mathrm{~mm}$ caffeine, and $100 \mathrm{~mm}$ salicin. $\boldsymbol{A}$, Total number of spikes generated over the initial 0.5 s of response. $\boldsymbol{B}$, Time-intensity curves (i.e., number of spikes during 5 consecutive $100 \mathrm{~ms}$ bins) from each class of bitter-sensitive taste cell; we present the curves elicited by aristolochic acid (left), caffeine (middle), and salicin (right) separately. All caterpillars were habituated to salicin before the recordings. Each bar or symbol indicates mean \pm SE. $n=10-18$ bitter-sensitive taste cells per sensillum, each from a different caterpillar.

Table 1. Predictions about whether the salicin-habituation phenomenon should generalize to caffeine and/or aristolochic acid, according to four sensory coding frameworks

\begin{tabular}{llllll}
\hline \multirow{2}{*}{$\begin{array}{lllll}\text { Coding } \\
\text { framework }\end{array}$} & \multicolumn{2}{l}{ Intact caterpillars } & & \multicolumn{2}{l}{ Lat-ablated caterpillars } \\
\cline { 2 - 3 } & Caffeine & Aristo acid & & Caffeine & Aristo acid \\
\hline Rate & - & - & - & - & + \\
Ensemble & + & - & & + & - \\
Temporal & + & - & & + & + \\
Spatiotemporal & - & - & & + & + \\
\hline
\end{tabular}

These predictions are based on analyses of the data in Figure 3 (see Results). We indicate that the salicin habituation should generalize to caffeine or aristolochic acid (Aristo acid) with a + , and that it should not generalize with a In addition, we show predictions for caterpillars possessing all taste sensilla (i.e., intact) and for those lacking their lateral styloconic sensilla (i.e., lat-ablated).

To test for distinct spatiotemporal codes, we ran two-way ANOVAs on the T-I curves from the two bitter-sensitive taste cells, separately for each taste stimulus. For each of the bitter taste stimuli, there was a nonsignificant interaction of taste cell $\times$ time (in all cases, $p>0.05$ ). This finding, together with visual inspection of Figure $3 b$, demonstrates that each bitter taste stimulus generated T-I curves in the different classes of bitter-sensitive taste cells that were effectively parallel to one another. This indicates that salicin, caffeine, and aristolochic acid all generated similar spatiotemporal codes in the lat-ablated caterpillars.

\section{Predictions for the pattern of stimulus generalization}

We used the results of the neural analyses described above to formulate predictions about whether the salicin-habituation should generalize to caffeine or aristolochic acid, according to each of the coding frameworks (Table 1). We formulated a separate set of predictions for intact and lat-ablated caterpillars. We reasoned that a match between the predictions of a particular coding framework and the pattern of stimulus generalization, in both intact and lat-ablated caterpillars, would provide compelling evidence that the caterpillars use that coding mechanism in discriminative taste processing.

Experiment 3: which coding framework best explains the pattern of stimulus generalization?

To assess the validity of the biting test, we ran a positive-control test with the intact nonhabituated caterpillars. We subjected each caterpillar to three brief-access biting tests. In each test, the experimental disk contained water (i.e., control stimulus), $0.03 \mathrm{~mm}$ aristolochic acid, or $5 \mathrm{~mm}$ caffeine. When the caterpillars were offered the control disk, their initial IBI was $\sim 0.5 \mathrm{~s}$ (Fig. 4, top left panel). When the same caterpillars were offered the caffeine or aristolochic acid disks, their initial IBI was significantly longer $(\sim 1.8 \mathrm{~s})$, indicating that the taste of the caffeine and aristolochic acid disks immediately inhibited biting. That this longer initial IBI reflects an aversive response is supported by the fact that biting activity across the initial $5 \mathrm{~s}$ of the test was significantly lower on the caffeine and aristolochic acid disks than on the control disk (Fig. 4, top right panel). These findings establish that we can detect an aversive response within the initial $0.5 \mathrm{~s}$ of the biting test, and both of the bitter taste stimuli elicit an aversive response in the absence of salicin habituation.

Next, we subjected the intact salicin-habituated caterpillars to the biting test. They exhibited similar biting responses to the control and caffeine disks. There was no difference in the initial IBI ( $\sim 0.5 \mathrm{~s}$ for both disks $)$ or in biting activity over the initial $5 \mathrm{~s}$ of the test (Fig. 4, bottom left panel). In contrast, the same caterpillars responded differently to the control and aristolochic acid disks. The initial IBI on the aristolochic acid disk $(\sim 1.9 \mathrm{~s})$ was significantly longer than that on the control disk, and biting activity was significantly lower (Fig. 4, bottom right panel). These findings establish that the salicin habituation generalized to caffeine but not to aristolochic acid. Additionally, given that the initial IBI on the control disk was $\sim 0.5 \mathrm{~s}$, and that the presence of aristolochic acid (but not caffeine) significantly increased the duration of the initial IBI, it follows that the operative time frame for the discrimination phenomenon was $\leq 0.5 \mathrm{~s}$.

Finally, we subjected the lat-ablated salicin-habituated caterpillars to the biting test. They displayed similar biting responses to the control and caffeine disks. There was no significant difference in the initial IBI $(\sim 0.5 \mathrm{~s}$ for both disks) or in biting activity over the initial $5 \mathrm{~s}$ of the test (Fig. 5). The same caterpillars responded quite differently to the control and aristolochic acid disks. The initial IBI on the aristolochic acid disk $(\sim 1.0 \mathrm{~s})$ was significantly longer than that on the control disk, and the caterpillars exhibited significantly lower biting activity on the aristolochic acid disk throughout the initial $5 \mathrm{~s}$ of the biting test (Fig. 5). These findings reveal that ablation of the lateral sensilla did not alter the pattern of stimulus generalization, that is, the salicin habituation still generalized to caffeine but not to aristolochic acid.

To assess the contribution of rate, ensemble, temporal, and 

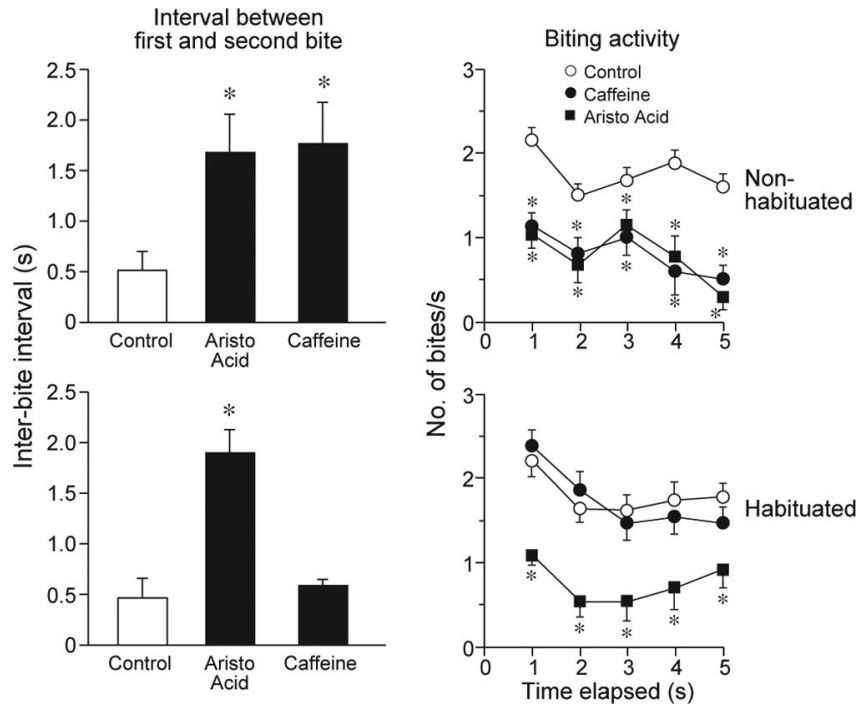

Figure 4. Initial biting responses of nonhabituated (top row) and salicin-habituated (bottom row) caterpillars to disks treated with water (i.e., control), $0.03 \mathrm{~mm}$ aristolochic acid (Aristo Acid), and $5 \mathrm{~mm}$ caffeine. In the left column, we show the initial interbite interval on each type of disk. In the right column, we show biting activity (i.e., number of bites $/ \mathrm{s}$ ) across the initial $5 \mathrm{~s}$ of the biting test. The test began once the caterpillar took its first bite. Within each panel, we compare the biting response to each bitter disk with that to the control disk, using the paired $t$ test $\left({ }^{*} p \leq 0.05\right.$, after Bonferroni correction). All values are mean $\pm \mathrm{SE} ; n=13$ nonhabituated and 18 salicin-habituated caterpillars.
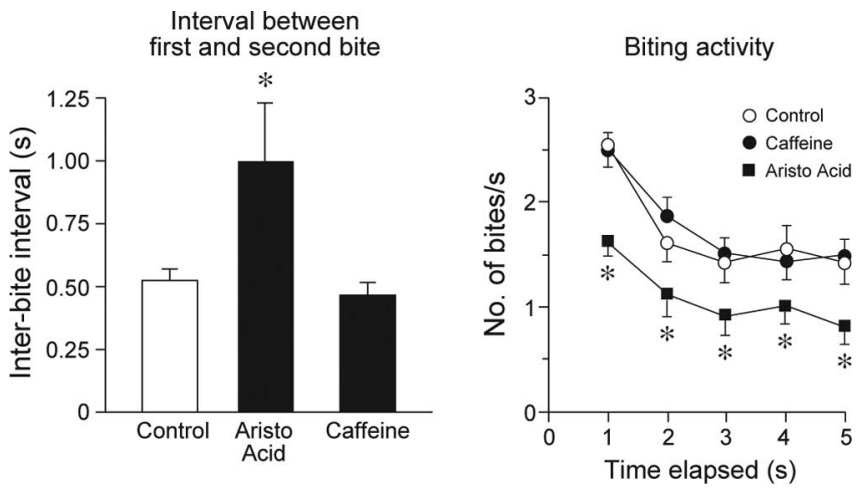

Figure 5. Initial biting responses of salicin-habituated caterpillars, lacking their lateral styloconic sensilla, to disks treated with water (i.e., control), $0.03 \mathrm{~mm}$ aristolochic acid (Aristo Acid), or $5 \mathrm{~mm}$ caffeine. In the left panel, we show the initial interbite interval on each type of disk. In the right panel, we show biting activity (i.e., number of bites/s) across the initial $5 \mathrm{~s}$ of the brief-access taste test. The test began once the caterpillar took its first bite. Within each panel, we compare the biting responses to each bitter disk with that to the control disk, using the paired $t$ test $\left({ }^{*} p \leq 0.05\right.$, after Bonferroni correction). All values are mean $\pm \mathrm{SE} ; n=22$ lat-ablated caterpillars.

spatiotemporal coding to the biting responses, we compared the results of the habituation-generalization tests with the predictions of each coding framework (Table 1). These comparisons revealed that the pattern of habituation generalization, in both intact and lat-ablated caterpillars, was consistent only with the predictions of the temporal coding framework.

\section{Discussion}

When the bitter-sensitive taste cells of $M$. sexta encounter caffeine, salicin, or aristolochic acid, they send volleys of spikes up the gustatory neuraxis. We sought to determine which features of these spike volleys are used in discriminative taste processing. To this end, we evaluated the ability of the four coding frameworks to predict the pattern of habituation generalization in both intact and lat-ablated caterpillars. We found that the pattern of habituation generalization could only be predicted by the temporal coding framework. As a result, we propose that aristolochic acid activated an aversive response in the salicin-habituated caterpillars because it elicited a discharge pattern that contrasted with the one elicited by the habituating diet. In contrast, caffeine failed to elicit an aversive response, because it elicited the same discharge pattern as the habituating diet.

Two findings are incompatible with a role of ensemble, spatiotemporal, or rate codes in the discrimination process. First, although caffeine and aristolochic acid generated similar ensemble and spatiotemporal codes, the lat-ablated caterpillars nevertheless discriminated between these two bitter taste stimuli. This indicates that distinct ensemble and spatiotemporal codes were not necessary for the discrimination. Second, we demonstrated elsewhere that the strength of the aversive response to caffeine and aristolochic acid increases with firing rate of the bittersensitive taste cells (Glendinning et al., 1999). Given that caffeine elicited a higher firing rate than aristolochic acid in the salicinhabituated caterpillars ( $p \leq 0.05$, according to a two-way ANOVA of the results in Fig. $3 a$ ), it follows that caffeine should have elicited a stronger aversive response than aristolochic acid, according to the rate coding framework. The fact that caffeine failed to elicit an aversive response altogether, and aristolochic acid elicited a robust aversive response, indicates that the salicinhabituated caterpillars ignored differences in firing rate.

Our claim of a causal connection between discharge patterns in the peripheral taste system and the pattern of stimulus generalization rests on two assumptions. The first is that the taste cell recordings accurately reflect the gustatory input that caterpillars experienced during the biting tests. We are confident about this assumption because we showed that the bitter-sensitive taste cells generated discriminable temporal codes within the same time frame as the discrimination phenomenon (i.e., in $\leq 0.5 \mathrm{~s}$ ). The second assumption is that the biting responses to the bitter taste stimuli were mediated by taste and not by other physiological mechanisms (e.g., aversive odor, tactile, and/or toxic effects). We are confident about this second assumption because we demonstrated previously that the aversive response of $M$. sexta to caffeine, aristolochic acid, and salicin is completely abolished by ablating the three classes of taste sensilla that respond to these taste stimuli (i.e., the lateral styloconic, medial styloconic, and epipharyngeal sensilla) (Glendinning et al., 1999).

Despite numerous reports of taste-specific discharge patterns in peripheral gustatory afferents (Ogawa et al., 1973, 1974; Nagai and Ueda, 1981; Dethier and Crnjar, 1982; Varkevisser et al., 2001) and central gustatory circuits (Di Lorenzo and Schwartzbaum, 1982; Katz et al., 2002b; Di Lorenzo and Victor, 2003; Verhagen and Scott, 2004), there have been comparatively few attempts to link discharge patterns to taste-guided behavioral responses. One series of experiments by Di Lorenzo and Hecht (1993) and Di Lorenzo et al. (2003) asked whether taste-specific discharge patterns from neurons in the nucleus of the solitary tract (NST; the first synaptic relay for taste in vertebrates) modulate taste-guided licking responses of rats. When they directly stimulated the NST with a train of pulses that mimicked the response to oral stimulation with quinine (a bitter taste stimulus), the rats terminated licking immediately. When they presented the same train of pulses, but with the timing of pulses randomized, the rats continued licking. This indicates that the quinine-specific discharge pattern was sufficient to mediate an aversive response. Our results extend these studies by showing: 
(1) insects, like rats, can use taste-specific discharge patterns in taste quality coding; and (2) taste-specific discharge patterns from taste cells can mediate discriminative taste processing.

Future studies are needed to determine the location and nature of the neural circuit(s) that discriminate between accelerating and decelerating discharge patterns. The most likely location is the subesophageal ganglion (SOG), which is the first synaptic relay in the gustatory neuraxis and a major site for the integration of orosensory input and oromotor output (Altman and Kien, 1987; Blaney and Simmonds, 1987; Kent and Hildebrand, 1987; Griss et al., 1991; Rohrbacher, 1994; Bowdan and Wyse, 2000). We can envision two neural mechanisms by which $M$. sexta could discriminate discharge patterns. First, there could be neural circuits in the SOG that discriminate between spike trains with gradually increasing versus decreasing interspike intervals. Second, M. sexta is known to have mechanosensory sensilla associated with its taste sensilla, which are bent (i.e., stimulated) whenever the taste sensilla are brought into contact with food (Devitt and Smith, 1985; Kent and Hildebrand, 1987). If M. sexta possessed neural circuits in the SOG that monitored the timing of inputs from adjacent mechanosensory and gustatory sensilla, then it could determine the discrepancy between the onsets of the tactile and gustatory input. Accordingly, the discrepancy would be greater when sampling a taste stimulus that elicits an accelerating discharge pattern (e.g., aristolochic acid) that when sampling one that elicits a decelerating discharge pattern (e.g., caffeine).

\section{Functional implications}

The use of temporal coding in discriminative taste processing should benefit M. sexta in several ways. First, compared with many insects [e.g., Orthopterans and Dipterans (Chapman et al., 1991; Meunier et al., 2003)], caterpillars have a paucity of bittersensitive taste cells, $\sim 16$ in total (Fig. 1 ). Such a small number of bitter-sensitive taste cells would limit the discriminatory potential of a spatial (i.e., labeled-line or ensemble) coding mechanism. Thus, the existence of a temporal coding mechanism would compliment existing spatial coding mechanisms in the taste system of M. sexta (Glendinning et al., 2002) and effectively increase the number of bitter taste stimuli that could be discriminated. Second, plants produce an incredible diversity of secondary compounds that taste bitter to humans and elicit an aversive response in other animal species. Although some of these bitter compounds are toxic, many are harmless (Bate-Smith, 1972; Garcia and Hankins, 1975; Brower, 1984; Brieskorn, 1990; Glendinning, 1994). We demonstrate herein that temporal coding enabled $M$. sexta to discriminate between bitter compounds that differ in toxicity [i.e., salicin and caffeine are relatively harmless, whereas aristolochic acid is toxic (Glendinning et al., 2001)]. This discriminatory ability should help M. sexta differentiate bitter plant compounds in their environment and learn to feed selectively on plants containing relatively harmless ones (Glendinning, 1994).

Finally, it has been suggested that animals cannot discriminate compounds that are detected by the same taste cells (Bernhardt et al., 1996; Adler et al., 2000; Dunipace et al., 2001). Our results contradict this suggestion. They show that M. sexta can discriminate compounds that are detected by the same bitter-sensitive taste cells, as long as the compounds generate distinct discharge patterns. Given that at least one other species of caterpillar (e.g., Bombyx mori) has bitter-sensitive taste cells that generate different discharge patterns in response to different plant compounds (Asaoka, 2000), it is likely that the discrimination phenomenon described herein is more taxonomically widespread.

\section{References}

Adler E, Hoon MA, Mueller KL, Chandrashekar J, Ryba NJP, Zuker CS (2000) A novel family of mammalian bitter taste receptors. Cell 100:693-702.

Altman JS, Kien J (1987) Functional organization of the subesophageal ganglion in arthropods. In: Arthropod brain: its evolution, development, structure and functions (Gupta AP, ed), pp 265-301. New York: John-Wiley.

Asaoka K (2000) Deficiency of gustatory sensitivity to some deterrent compounds in "polyphagous" mutant strains of the silkworm, Bombyx mori. J Comp Physiol A Neuroethol Sens Neural Behav Physiol 186:1011-1018.

Bate-Smith EC (1972) Attractants and repellents in higher animals. In: Phytochemical ecology: proceedings of the phytochemical society symposium (Harborne JB, ed), pp 45-56. London: Academic.

Bell RA, Joachim FA (1976) Techniques for rearing laboratory colonies of tobacco hornworms and pink bollworms. Ann Entomol Soc Am 69:365-373.

Bernays EA, Chapman RF (1994) Host-plant selection by phytophagous insects. New York: Chapman and Hall.

Bernays EA, Chapman RF, Singer MS (2000) Sensitivity to chemically diverse phagostimulants in a single gustatory neuron of a polyphagous caterpillar. J Comp Physiol A Neuroethol Sens Neural Behav Physiol 186:13-19.

Bernhardt SJ, Naim M, Zehavi U, Lindemann B (1996) Changes in $\mathrm{IP}_{3}$ and cytosolic $\mathrm{Ca}^{2+}$ in response to sugars and non-sugar sweeteners in transduction of sweet taste in the rat. J Physiol (Lond) 490:325-336.

Blaney WM, Simmonds MSJ (1987) Experience: a modifier of neural and behavioural sensitivity. In: Insects-plants (Labeyrie V, Fabres G, Lachaise D, eds), pp 237-241. Dordrecht, The Netherlands: Dr. W. Junk Publishers.

Bowdan E, Wyse GA (2000) Temporally patterned activity recorded from mandibular nerves of the isolated subesophageal ganglion of Manduca. J Insect Physiol 46:709-719.

Brieskorn CH (1990) Physiological and therapeutic aspects of bitter compounds. In: Bitterness in foods and beverages (Rouseff RL, ed), pp 15-33. New York: Elsevier.

Brower LP (1984) Chemical defense in butterflies. In: The biology of butterflies (Vane-Wright RI, Ackery PR, eds), pp 109-134. London: Academic.

Caicedo A, Kim K-N, Roper SD (2002) Individual mouse taste cells respond to multiple chemical stimuli. J Physiol (Lond) 544:501-509.

Chapman RF, Ascoli-Christensen A, White PR (1991) Sensory coding for feeding deterrence in the grasshopper Schistocerca americana. J Exp Biol 158:241-259.

de Boer G, Hanson FE (1987) Differentiation of roles of chemosensory organs in food discrimination among host and non-host plants by larvae of the tobacco hornworm, Manduca sexta. Physiol Entomol 12:387-398.

de Boer G, Dethier VG, Schoonhoven LM (1977) Chemoreceptors in the preoral cavity of the tobacco hornworm, Manduca sexta, and their possible function in feeding behavior. Entomol Exp Appl 21:287-298.

Dethier VG, Crnjar RM (1982) Candidate codes in the gustatory system of caterpillars. J Gen Physiol 79:549-569.

Devitt BD, Smith JJB (1985) Action of mouthparts during feeding in the dark-sided cutworm, Euoxa messoria (Lepidoptera: Noctuidae). Can Entomol 117:343-349.

Di Lorenzo P, Hecht GS (1993) Perceptual consequences of electrical stimulation in the gustatory system. Behav Neurosci 107:130-138.

Di Lorenzo P, Hallick RM, Kennedy DP (2003) Temporal coding of sensation: mimicking taste quality with electrical stimulation of the brain. Behav Neurosci 117:1423-1433.

Di Lorenzo PM, Schwartzbaum JS (1982) Coding of gustatory information in the pontine parabrachial nuclei of the rabbit: temporal patterns of neural response. Brain Res 251:245-257.

Di Lorenzo PM, Victor JD (2003) Taste response variability and temporal coding in the nucleus of the solitary tract of the rat. J Neurophysiol 90:1418-1431.

Dunipace L, Meister S, McNealy C, Amrein H (2001) Spatially restricted expression of candidate taste receptors in the Drosophila gustatory system. Curr Biol 11:822-835.

Erickson RP, Schiffman SS, Doetsch GS, Di Lorenzo PM, Woodbury MA (1995) A fuzzy set approach to the organization of the gustatory system. Primary Sensory Neuron 1:65-80.

Frazier JL (1986) The perception of plant allelochemicals that inhibit feed- 
ing. In: Molecular aspects of insect-plant interactions (Brattsten LB, Ahmad S, eds), pp 1-42. New York: Plenum.

Garcia J, Hankins WG (1975) The evolution of bitter and the acquisition of toxiphobia. In: Olfaction and taste. V. Proceedings of the 5th international symposium in Melbourne, Australia (Denton DA, Coghlan JP, eds), pp 39-45. New York: Academic.

Glendinning JI (1994) Is the bitter rejection response always adaptive? Physiol Behav 56:1217-1227.

Glendinning JI, Hills TT (1997) Electrophysiological evidence for two transduction pathways within a bitter-sensitive taste receptor. J Neurophysiol 78:734-745.

Glendinning JI, Valcic S, Timmermann BN (1998) Maxillary palps can mediate taste rejection of plant allelochemicals by caterpillars. J Comp Physiol A Neuroethol Sens Neural Behav Physiol 183:35-44.

Glendinning JI, Tarre M, Asaoka K (1999) Contribution of different bittersensitive taste cells to feeding inhibition in a caterpillar (Manduca sexta). Behav Neurosci 113:840-854.

Glendinning JI, Nelson N, Bernays EA (2000) How do inositol and glucose modulate feeding in Manduca sexta caterpillars? J Exp Biol 203:1299-1315.

Glendinning JI, Domdom S, Long E (2001) Selective adaptation to noxious foods by an insect. J Exp Biol 204:3355-3367.

Glendinning JI, Davis A, Ramaswamy S (2002) Contribution of different taste cells and signaling pathways to the discrimination of "bitter" taste stimuli by an insect. J Neurosci 22:7281-7287.

Gothilf S, Hanson FE (1994) A technique for electrophysiologically recording from chemosensory organs of intact caterpillars. Entomol Exp Appl 72:304-310.

Griss C, Simpson SJ, Rohrbacher J, Rowell CHF (1991) Localization in the central nervous system of larval Manduca sexta (Lepidoptera: Sphingidae) of areas responsible for aspects of feeding behavior. J Insect Physiol 37:477-482.

Hellekant G, Danilova V, Ninomiya Y (1997) Primate sense of taste: behavioral and single chorda tympani and glossopharyngeal nerve fiber recordings in the rhesus monkey, Macaca mulatta. J Neurophysiol 77:978-993.

Hodgson ES, Lettvin JY, Roeder KD (1955) Physiology of a primary chemoreceptor unit. Science 122:417-418.

Katz DB (2005) The many flavors of temporal coding in the gustatory cortex. Chemical Senses 30 [Suppl 1]:80-81.

Katz DB, Nicolelis MA, Simon SA (2002a) Gustatory processing is dynamic and distributed. Curr Opin Neurobiol 12:448-454.

Katz DB, Simon SA, Nicolelis MA (2002b) Taste-specific neuronal ensembles in the gustatory cortex of awake rats. J Neurosci 22:1850-1857.
Kent KS, Hildebrand JG (1987) Cephalic sensory pathways in the central nervous system of larval Manduca sexta (Lepidoptera: Sphingidae). Philos Trans R Soc Lond B Biol Sci 315:1-36.

Marella S, Fischler W, Kong P, Asgarian E, Scott K (2006) Imaging taste responses in the fly brain reveals a functional map of taste category and behavior. Neuron 49:285-295.

Marion-Poll F, Van der Pers J (1996) Un-filtered recordings from insect taste sensilla. Entomol Exp Appl 80:113-115.

Meunier N, Marion-Poll F, Rospars JP, Tanimura T (2003) Peripheral coding of bitter taste in Drosophila. J Neurobiol 56:139-152.

Nagai T, Ueda K (1981) Stochastic properties of gustatory impulse discharges in rat chorda tympani fibers. J Neurophysiol 45:574-593.

Ogawa H, Sato M, Yamashita S (1973) Variability in impulse discharges in rat chorda tympani fibers in response to repeated gustatory stimulations. Physiol Behav 11:469-479.

Ogawa H, Yamashita S, Sato M (1974) Variation in gustatory nerve fiber discharge pattern with change in stimulus concentration and quality. J Neurophysiol 37:443-457.

Rohrbacher J (1994) Fictive chewing activity in motor neurons and interneurons of the subesophageal ganglion of Manduca sexta larvae. J Comp Physiol A Neuroethol Sens Neural Behav Physiol 175:629-637.

Schoonhoven LM (1972) Plant recognition by lepidopterous larvae. In: Insect/plant relationships (van Emden HF, ed), pp 87-99. Oxford: Blackwell Scientific.

Scott TR, Giza BK (1987) A measure of taste intensity discrimination in the rat through conditioned taste aversions. Physiol Behav 41:315-320.

Scott TR, Perrotto RS (1980) Intensity coding in pontine taste area: gustatory information is processed similarly throughout rat's brain stem. J Neurophysiol 44:739-750.

Städler E, Hanson FE (1975) Olfactory capabilities of the "gustatory" chemoreceptors of the tobacco hornworm. J Comp Physiol 104:97-102.

Varkevisser B, Peterson D, Ogura T, Kinnamon S (2001) Neural networks distinguish between taste qualities based on receptor cell population responses. Chem Sens 26:499-505.

Verhagen JV, Scott TR (2004) Artificial neural network analysis of gustatory responses in the thalamic taste relay of the rat. Physiol Behav 80:499-513.

White PR, Chapman RF, Ascoli-Christensen A (1990) Interactions between two neurons in contact chemosensilla of the grasshopper, Schistocerca americana. J Comp Physiol A Neuroethol Sens Neural Behav Physiol 167:431-436.

Zhang Y, Hoon MA, Chandrashekar J, Mueller KL, Cook B, Wu D, Zuker CS, Ryba NJ (2003) Coding of sweet, bitter, and umami tastes: different receptor cells sharing similar signaling pathways. Cell 112:293-301. 